\title{
Shaping the Future of Smart Dentistry: From Artificial Intelligence (AI) to Intelligence Augmentation (IA)
}

\author{
Hossein Hassani ${ }^{1, *}$, Pedram Amiri Andi ${ }^{2}$, Alireza Ghodsi ${ }^{2}$, Kimia Norouzi ${ }^{2}$, Nadejda Komendantova ${ }^{3}$ (i) \\ and Stephan Unger 4 \\ 1 Research Institute of Energy Management and Planning (RIEMP), University of Tehran, \\ Tehran 1417466191, Iran \\ 2 Faculty of Dentistry, Tehran Medical Sciences, Islamic Azad University, Dental Branch, Pasdaran Street, \\ Tehran 1946853314, Iran; pedramamiriandi@dentment.ir (P.A.A.); Dralirezaghodsi@dentment.ir (A.G.); \\ kimianorouzi@dentment.ir (K.N.) \\ 3 International Institute for Applied Systems Analysis (IIASA), A-2361 Laxenburg, Austria; \\ komendan@iiasa.ac.at \\ 4 Department of Economics and Business, Saint Anselm College, Manchester, NH 03102, USA; \\ sunger@anselm.edu \\ * Correspondence: hosseinhassani57@webster.edu
}

Citation: Hassani, H.; Amiri Andi, P.; Ghodsi, A.; Norouzi, K.;

Komendantova, N.; Unger, S. Shaping the Future of Smart Dentistry: From Artificial Intelligence (AI) to Intelligence Augmentation (IA). IoT 2021, 2, 510-523. https:// doi.org/10.3390/iot2030026

Academic Editor: Hyun-Ho Choi

Received: 7 August 2021

Accepted: 25 August 2021

Published: 30 August 2021

Publisher's Note: MDPI stays neutral with regard to jurisdictional claims in published maps and institutional affiliations.

\begin{abstract}
Digitization is the emerging process in the current transformation of industry. Understanding the role and socio-economic consequences of digitalization is crucial for the way technology is being deployed in each sector. One of the affected sectors is dentistry. This study highlights the current advances and challenges in integrating and merging artificial intelligence (AI), intelligence augmentation (IA), and machine learning (ML) in dentistry. We conduct a comparative analysis to give an overview of which technology is being currently deployed and what role IA and AI will play in dentistry, as AI plays an assistive role in advancing human capabilities. We find that challenges range from AI finding its way into routine medical practice to qualitative challenges of retrieving adequate data input. Other challenges lie in the yet unanswered questions of liability in how to reduce deployment costs of new technology. Given these challenges, we provide an outlook of how future technology can be deployed in daily-life dentistry and how robots and humans will interact, given the current technology developments. The aim of this paper is to discuss the future of dentistry and whether it is AI or IA conquering the modern dentistry era.
\end{abstract}

Keywords: dentistry; smart dentistry; artificial intelligence (AI); intelligence augmentation (IA)

\section{Introduction}

The 21st century has become a century of new innovative technologies and processes, such as digitalization and deployment of industry 4.0. Digital technologies have started playing an important role in every aspect of our daily lives. They already have a great influence on nearly all existing industries, and their impact continues to grow. The dentistry sector is the economic sector that might benefit significantly from introduction of digital technologies. The proper use of potential technologies is also required in dentistry to provide an efficient treatment system, enabling not only the dentist and patients but also the dentistry students in saving much time and money, plus decreasing human mistakes.

According to the World Dental Federation [1], globally 3.9 billion people are affected by oral diseases and untreated tooth decay impacts nearly half of the world's population; $44 \%$ are suffering from the untreated impacts of tooth decay. This makes it the most common disease amongst all the 291 conditions contained within the Global Burden of Disease study. Considering these numbers, further implementations of digital technologies that increase efficiency in the dentistry sector seems to be fruitful. In addition, being considered the fourth-most expensive disease to treat, oral diseases make the matter of 
investment a remarkable issue. Besides, associating with significant pain and anxiety, deformities, acute or chronic infections, eating and sleeping disturbances, etc., oral diseases can impact every aspect of life, such as relationships and self-confidence, and result in unsociability and reduced income, leading to a low-quality life generally. Additionally, oral health plays an essential role in maintaining general health, making it an extremely crucial field to consider.

The first technologies that increase efficiency in the dentistry sector were implemented in 1092. Starting from this year, the barber surgeons arose. Subsequent technological advances, including the invention of amalgam as a material for tooth restoration and dentures to replace missing teeth, which were made of ivory, hippopotamus or human bone, or metal in the mid-19th century, a major advancement in caries treatment, the use of dental drills became a major breakthrough in speeding up removal of decayed tissue. In addition, advances in dental education have been tooled over the centuries [2].

Today the use of technologies in dentistry can be seen in computer-assisted design and computer-assisted manufacture (CAD/CAM) and intraoral imaging-both laboratory and clinician controlled. The technologies are used in caries diagnosis, in computer-aided implant dentistry, including the design and fabrication of surgical guides; in digital radiography (intraoral and extraoral), including cone beam computed tomography (CBCT); in electric and surgical/implant handpieces, lasers, occlusion, and temporomandibular joint (TMJ) analysis and diagnosis; in photography (extraoral and intraoral); in practice and patient record management, including digital patient education; and in shade matching [2,3]. With all these digital dentistry implements helping dentistry worldwide, researchers and inventors are further stimulated to continuously seek for new technologies or advancements that can be used to elevate the profession to its optimal state.

A thought-provoking idea seemingly enhancing the future of dentistry is the use of the AI concept [4], which was given by John McCarty in 1956. Besides, there is another concept, IA, which is parallel to AI and was constructed by Engelbart [5]. In this paper, we aim to investigate the future of smart dentistry and discuss whether it is AI or IA conquering the modern dentistry era.

The contribution of our study to the existing literature is the enhancement of conducted studies by working out how various AI methods are being deployed in dentistry and analyzing the effect of these technologies in this sector. Current research mainly takes one specific technology and analyzes its deployment, challenges, and impact in dentistry, whereas we provide a comprehensive overview of the different technologies, their status quo, and potential, as well as challenges linked to their implementation. Our motivation lies in the current rapid deployment of digitization in all industry sectors. Therefore, we think it is important to provide a comprehensive oversight of the major developments in artificial intelligence, applied to dentistry, as well as the associated challenges that come with it.

Our paper is structured as follows: In Section 2, we provide a general overview about artificial intelligence and intelligence augmentation and their application in dentistry. In Section 3, we outline current advances and stress challenges in AI methods in dentistry. Section 4 provides examples of intelligent machines in dentistry, and Section 5 tackles the data privacy issues and security approaches in smart dentistry. Section 6 outlines the prospects and future investment potential of AI technology in dentistry, while Section 7 stresses the obstacles and limitations of robotic dentistry. In Section 8, we propose future research issues and conclude in Section 9.

\section{Artificial Intelligence vs. Intelligence Augmentation}

Coming up with AI theory, its father John McCarty defined AI as "the science and engineering of making intelligent machines" [6].

$\mathrm{AI}$ is inspired from human intelligence that has been defined by many conceptualists as the ability that becomes practical when we do not know what to do or a general mental quality consisting of the abilities to learn from experiences, adapting to unexpected 
situations, understanding and handling deep concepts, using knowledge to interfere with one's environment, and reasoning and being able to learn tons of information [4-9].

In fact, AI is a machine's ability to mimic humans' manners and to take part in tasks normally adopted by intelligent beings, mining and aggregating huge datasets to provide more precise information in less time [10].

AI is composed of a set of algorithms collaborating in line with human beings. Its functioning is based on a set of linked units or nodes creating a computing system roughly patterned after the human brain, called artificial neural networks (ANNs).

A type of AI is explained when computer scientists fuel those algorithms by particular data supplements, defining some exact rules. The algorithms master the pattern lying in that information to provide an accurate certain answer. These mechanisms are simply titled "machine learning" (ML) [4,11,12].

Just as ML is a type of AI, so is deep learning a subtype of ML by lining up the artificial neurons in several layers. Linking those layers by complex mathematical processes, a network relatively capable of mimicking humans brain structure is formed, also known as the vanguard of ML; yet unlike it, deep learning machines do not need a human guide to construct them with the data. Processing and arranging a huge amount of random unstructured datasets gathered from every corner of the world, simply known as big data, would take humans several decades to perceive and unravel, while deep learning has great potential to extract relevant information and then create patterns itself for making rational decisions; thereby, the fuel for deep learning models is data as well [13,14].

Clearly, AI still has a long way to be in the same league as the human mind, since intelligence is an inherent characteristic of human beings and is still being tested to break its limits. It is unlikely for AI will be comparable with the human brain, since it has been formed by humans themselves who still do not know enough about their own brain $[5,15]$. That is why the brand-new alternative concept IA has recently been proposed. Instead of targeting the replacement of humans with machines, a number of researchers and conceptualists evoked the idea of using machines to work alongside the human mind, with the intention of augmenting and amplifying the human brain, allowing it to perform more efficiently in multiple fields [16]. In other words, IA can be classified as the creation of a mind tool [5]. The theory suggests merging technologies and the human mind in a way that enables humans to control and deploy machines to gain profits from them. In addition, even now, many applied technologies known as AI need to be categorized as IA.

Although the basic technologies used for both AI and IA are the same, IA works by putting humans at the focal point and machines serve them to fulfill their ambitions, but AI places technology first and tends to manufacture independent machines. A brief table below (Table 1) summarizes the different implementations of AI vs. IA using the specific example of dentistry.

Table 1. AI vs. IA in dentistry.

\begin{tabular}{ccc}
\hline AI in Dentistry & IA in Dentistry \\
\hline Implementation & $\begin{array}{c}\text { Technology-empowered } \\
\text { robots/systems working } \\
\text { platforms replacing dentists }\end{array}$ & $\begin{array}{c}\text { collaboratively with dentists on } \\
\text { complex tasks, improving accuracy } \\
\text { and efficiency }\end{array}$ \\
\hline
\end{tabular}

\section{Advances vs. Challenges}

Various AI techniques have already been applied in dentistry. Among those that are most widely spread are deep learning and artificial learning networks, Bayesian networks, convolutional neural networks, support vector machines, logistic regressions, and decision trees. Chen et al. [8] provide a good overview of current applications and future perspectives of $\mathrm{AI}$ in dentistry. 
In the area of radiology, Lee et al. [17] used a deep learning network for the diagnosis of caries. Jung and Kim [18] used an artificial neural network for the diagnosis of the need for orthodontic extraction. Neural networks try to minimize the forecasting error between target function and input, e.g., a radiographic image of a tooth. Then the neural network is trained on the image for how a tooth should look like, thus relying on statistical patterns. The data points that are generated during the training process are assigned labels (classification task), or numerical results (regression task) are repetitively passed through the neural network. The key of a neural network is that the model weights are updated in each repetition and iteratively converge to a minimal forecasting error, where the forecasting error is nothing other than the difference between the true and the predicted result. The strength of a neural network that is trained lies in its ability to predict unknown data or patterns by passing new data through itself.

Furthermore, in the area of orthodontics, Thanathornwong [19] applied Bayesian networks for AI clinical decision support, while Patcas et al. [20] made use of a convolutional neural network to conduct treatment outcome analysis. In the field of periodontics, Lee et al. [17] used convolutional neural networks as well for the diagnosis and prediction of periodontally compromised teeth. A convolutional neural network is a deep learning neural network that is specifically designed to process pixel data. A different way to analyze data for classification and regression analysis is the use of support vector machines. Feres et al. [21] applied a support vector machine in periodontics for the differentiation between aggressive and chronic periodontitis. In oral medicine, artificial neural networks, support vector machines, logistic regressions, and decision tress were used by Kim et al. [22] for the prediction of MRONJ and by Speight et al. [23] for the risk assessment of oral cancer.

Schwendicke et al. [24] give an overview of the chances and challenges in AI in dentistry. They highlight the importance of diagnostic imaging in healthcare and the effective deployment of AI to lower variability in examination, while lowering the costs by eliminating routine tasks. Moreover, they mention the ability of AI to integrate heterogeneous data, which is extremely useful when it comes to adding experimentation options. However, they also highlight the challenges that come along with AI, since it has not found its way into routine medical practice yet. Another challenge concerns the replicability of results, since it is often unclear how datasets were selected, curated, and preprocessed.

So, the challenges in the application of AI in dentistry are manifold. What is known is that the output can only be as good as the input. That means that regardless of which AI technique is being used, the source of the data needs to be better understood in order to find the roots of the problem. Without a proper understanding of causality and linkages between origination of a disease or malfunction, the AI system can only conduct a symptomatic analysis, which means that the treatment will also be symptomatic.

Nevertheless, this gap is likely to be narrowed with time as increasingly more data are being generated and made available to the AI systems to learn.

Another challenge with AI-applied dentistry is related to the usage and creation of data. The corresponding arising privacy concerns are limited to not only dentistry but health data in general. As increasingly more dental cases are being processed, increasingly more data are being generated. On the one hand, this is beneficial to the progress and enhancement of dental AI performance, as with increasing data to learn from, the performance of any AI system increases. On the other hand, the question about which dental data can or should be used for re-processing, with respect to privacy, is an ethical issue that will only continue to grow with increasing deployment of AI machinery in dentistry.

Schwendicke et al. [24] mention looming challenges because of which AI solutions have not, by large, entered routine dental practice: (1) limited data availability, accessibility, structure, and comprehensiveness; (2) a lack of methodological rigor and standards in their development; (3) and practical questions around the value and usefulness of these solutions. They also mention the problems associated with ethics, individual privacy rights, and autonomy, as well as the importance of continuous human oversight and standards grounded in evidence-based dentistry. 
Dhelim et al. [25] propagate the consideration of the social properties and attributions of Internet of Things (IoT) entities through the application of artificial social intelligence. The management of social relationships in IoT, including social-oriented machine learning and deep learning techniques, could help in the networking process of doctors and researchers in dentistry for diagnostics, research, and treatment. Nevertheless, they also stress the importance of tackling existing bottlenecks, such as in computing and communication.

Cai et al. [26] set up a theoretical framework of a robot's living space to further understand the human-robot relationship. Their research shows the importance of an appropriate work space environment of robots in dentistry and how to make a collaboration with intelligent robots to achieve hybrid intelligence as well as to build a harmonious human-robot-integrated society.

A big upcoming challenge concerning an ethical issue will be the question of triage with respect to emergency situations, e.g., during a surgery, when a robot faces a situation in which any out of two possibilities will cause long-term damage to the patient but cannot be prevented, and has to decide instantaneously what to do.

Coming along with such challenges will be the question of liability for cases in which the robot might not conduct proper work and harm the patient. Other issues might arise out of a wrong diagnosis or a wrongly chosen treatment by the AI system.

Upcoming issues like these will lead to future regulation in robotics and AI, and therefore the AI dentistry sector, to evolve, including the emergence of new institutions concerned with the legal and ethical aspects of $\mathrm{AI}$ in the health industry. Moreover, the increasing need, advantages, and benefits of technological development in AI dentistry will lead to increased competition in this market, as AI systems become more reliable and cheaper to deploy. This will cause quality to equilibrate at a certain market standard with respect to costs.

The issues mentioned above lead to the conclusion that AI in dentistry will lead to increased deployment of IA for dental doctors, especially considering the technological progress that will allow one to process increasingly more data at an increasingly faster pace. The IA aspect of dentistry will lead to a pure supervision role of dental medicals, whose job will be the supervision and control of robots who will perform increasingly more complex routines.

A significant step will be the deployment of robots, especially when it comes to direct interaction with the patient. There is a difference between using robots for routine jobs, such as the scanning and 3D printing of crowns, but when it comes to the actual medical procedure, IA is likely to prevail for the longest foreseeable future. This means that the medical professional will still be in charge of the actual surgery, whereas the surgeon might not even need to be in the same room, as through $5 \mathrm{G}$ and real-time communication enabling, medical procedures will be possible even remotely.

\section{Essential Role of Intelligent Machines in Dentistry}

Progressing in collaboration with intelligent machines and modern technologies, dentistry seems to have an optimistic future ahead.

Mastering the proper use of technologies reduces the pressure on the dentist in office and patient management, including more effective treatment plans implemented due to the AI ability to explore entire patient records and X-rays quickly and providing more efficient reliable care. This is naturally followed by cost and time reduction. As expected, such technology also strengthens personalized oral healthcare that relies on the clinical and genetical characteristic of each patient [27]. Therefore, there is an assumption that if provided, such technology can lead to a great revolution in the dentistry treatment field. According to Dr. Vidone, who is the chief clinical officer at the Delta Dental of Massachusetts, the list of areas where AI can improve performance in the dentistry industry is long and includes diagnosis, treatment, prosthetics, practice management, and claims review. 


\subsection{Introducing New Waves of Technology}

\subsubsection{Pearl's Practice Intelligence Platform}

Considering that oral diseases are one of the most common ailments, sometimes dealing with all kinds of patients and managing them properly can be onerous for the clinicians. Pearl's Practice Intelligence is a platform that uses computer vision software to drill down to office-specific data that matter, providing highly specialized data, and makes it possible for practitioners to manage patients, make correct decisions, and come up with proper treatment plans [28].

\subsubsection{AI-Empowered Radiograph Diagnostics}

Despite the great impact of artificial intelligence on the development of imaging processes in dentistry, there is still a huge probability of failure and imprecise diagnosis of dental radiographs, an issue that can lead to the patient's distrust and pose a threat to the dental profession; this is where once again artificial intelligence can help.

In this regard, a number of new-found technologies with absolutely influential traits have been announced. These platforms work by collecting and annotating countless radiographic data provided by clinicians all around the world and then training AI models on these classified data to empower them in identifying these features in the new data so that the models can detect dozens of common pathologies accurately and quickly, including Pearl's Second Opinion, Denti.AI, and Videa Detect [28,29].

\subsubsection{VEL Scope}

As the eighth-most common cancer in the world, oral cancer is a tormenting phenomenon, and the early diagnosis of cancerous lesions increases the likelihood of cure and the survival chance.

A visual enhanced lesion (VEL) scope is a light-based oral-cancer-screening device that can help to detect oral cancer and mucosal lesions in early stages. This device can be either wired or wireless. It illuminates the epithelium by beaming a blue light $(400-460 \mathrm{~nm})$ on it; the healthy epithelium appears in a light-green color, and the damaged areas look darker as a result of losing their natural fluorescence. The usage of the VEL scope does not cause pain or irritation for the patient, and the process can be completed in a couple of minutes. The device also has the ability to easily connect to cameras or simply an iPad, enabling dentists to collect and access patient records with much less trouble.

However, the VEL scope also has some limitations according to [30]. Although the VEL scope aids skillfully in finding abnormalities that are invisible to the naked eye, it cannot decide whether a lesion is cancerous. It also has a high percentage of false-positive responses, which leads to the patient's reasonless concern.

\subsubsection{Dental Caries Diagnostics}

Dental caries is the most prevalent oral disease. It is a painful, chronic infectious disease caused by streptococcus mutants producing acid from food. Advanced caries can affect the pulp and pre-apical area and can cause pain and discomfort or even tooth loss for the patient.

A variety of devices have been introduced to help dentists to detect caries that is too small or sometimes inaccessible to conventional diagnostic tools, enabling clinicians to identify occlusal decays more efficiently. An example is the DIAGOdent laser system, where the DIAGOdent pen is a laser fluorescence system employing a laser diode that generates a pulsed $655 \mathrm{~nm}$ laser beam using a central fiber. It measures laser fluorescence within the mineral structure of the tooth. At that specific wavelength, the DIAGOdent system employs sound tooth structures, which show little or no fluorescence at all. Hence, low scale readings appear on the display, while the decayed tooth structure elevates the scale readings on the display due to exhibiting fluorescence comparable with the lost tooth structure degree. The operator can simply hear the changes in the scale values through an 
audio signal that eliminates the need for the dentist to constantly look at the screen, which increases the dentist's focus on the teeth [31].

\subsubsection{Air Abrasion}

Many people are afraid of dentist visits, which is known as dental fear, dental anxiety, dentist phobia, odontophobia, or dentophobia [32]. There are several reasons for this, but one of them might be the fear of dental drills. In an interview to the Guardians, Dr. Kabibe mentions, "As a pediatric dentist, I've seen many patients since 1987, and from my clinical experience, I found that the sound of drilling can evoke anxiety in dental patients" [33]. Air abrasion, which is also called microabrasion, is an alternative to drills. Air abrasion allows an extremely conservative preparation to maintain the maximum intact tooth structure, removing tooth decay using tiny particles of aluminum oxide or silica. It uses compressed air or bottled carbon dioxide or nitrogen gas, spraying a stream of sand-like particles with high velocity toward the damaged tooth and removing small amounts of the tooth structure; the excess must be suctioned away from the patient's mouth [34-36].

\subsubsection{Precise Physiological Reinstation of Occlusion}

Precise physiological reinstation of occlusion is important for a healthy functioning masticatory system. A vast variety of dental occlusion is seen among patients. It differs in tooth size, shape, and position, as well as in the stages of eruption timing and dental arch size. Unlike articulating paper, which can only determine location, the T-Scan system records the center of force, first contact, maximum bite force, and maximum intercuspation. It is a digital occlusion analysis system that measures tooth contact, force, and timing and then records them. It uses a thin, flexible, pressure-sensitive bite transducer implanted in a dental-arch-shaped recording sensor [37].

\subsubsection{Robots}

Robots represent the ultimate level of technological advancement in many industries today. Due to their ability to work with high precision indefatigably, robots are employed in dentistry as well, especially in minimally invasive dentistry. They offer a variety of services in dentistry; here are some of them.

\section{Dental Patient Robot}

Dental patient robots are a type of artificial creatures that are designed to mimic multiple humans' demeanors. Therefore, they are used as alternative study cases besides real patients, and dentistry students can benefit from them to master their clinical skills. Such robots are also known as phantoms.

Showa Hanako, for example, is a realistic patient robot created in Tokyo, Japan, by Showa University with assistance from the robotics company Tmsuk [38]. It has the ability to simulate a number of humans' natural reactions, such as blinking, sneezing, and coughing. It is also capable of shaking its head, rolling its eyes, and moving its tongue; more interestingly, it represents fatigue by keeping his mouth open for a while and also simulates the gag reflex, quite similar to humans [39].

In addition, there is Simroid, a more advanced example of such robots, created at the Nippon Dental University Kokoro. Including a built-in sensor, it expresses pain and discomfort in reaction to treatment procedures, its voice recognition system is practical for trainees to develop their communication skills as well, and it responds to the student's speech. It also has a realistic appearance, namely its skin can be torn while the mouth is wide open, in addition to other absolute natural features, such as facial expressions, hands and legs, and other natural body movements in general, which, it is said, sometimes makes it hard for practitioners to believe they are not dealing with a real human.

To record the full treatment session, this groundbreaking pseudo-human robot patient is equipped with two charge-coupled device (CCD) cameras. A monitor is also equipped 
for live monitoring of the procedure while recording it for later reviews. This allows for adequate posture adjustment, and the students are fully prepared for their real sessions [40].

\section{Endo Micro-Robot}

The endo micro-robot is capable of automatic probing, drilling, cleaning, shaping, and filling of the root canal; minimizes human errors; and eliminates the limitations of conventional methods, such as inadequate opening or exorbitance removal of the tooth structure, in order to provide accurate proper root canal treatment (RCT) [41].

\section{Implant Robot}

Implant robot surgery involves many risks, such as injury to the maxillary sinus, inferior alveolar canal, or labial or lingual plate due to perforation, nerve injury, tissue necrosis leading to dehiscence, or infection, which is why about $5 \%$ to $10 \%$ of dental implants meet with failure, which is also a risk itself.

A navigational robotic assistance system is offered by Neocis to help clinicians through the operation.

\subsubsection{AI Tooth Brushes}

Oral-B recently launched its $\$ 220$ Oral-B Genius X electric toothbrush. This brush is equipped with AI systems to give feedback on how effective or harmful one's brushing method is. The brush simply connects to an app on the phone via Bluetooth that shows how long the person has been brushing. It also provides guidance on the way someone brushes. In addition, it provides a warning about putting extra pressure while brushing or if someone is brushing some spot insufficiently or not long enough. It can also tell what should be brushed the next time. The brush uses some sensors to collect data from the mouth and send these data to the app, which evaluates the brushing technique using the Genius X AI algorithm [42].

\subsubsection{Planmeca Emerald}

Planmeca Emerald is an incredibly fast, weightless, easily carried intraoral scanner that allows full arch scanning in less than a few minutes. Due to its small size and ergonomic structure, it is more pleasant to use for patients. It also puts minimal pressure on the dentist's wrist. There are several other advantages of this technology over traditional impressions presented in this device, namely (a) digital accuracy that produces vivid colors accompanied by spotless details, (b) single appointment and short scanning sessions due to its high speed, and (c) flexible import and export, making digital impressions captured easy to share with workmates either in or outside the clinic $[9,43,44]$.

\section{Data Privacy and Security Approaches in Smart Dentistry}

One of the biggest issues with increasing digitization and linkages of AI with human interfaces is the question of data privacy and data security. Especially in the medical field, data are highly sensitive and they might have a heavy influence, e.g., on insurance premiums of clients if insurance companies are able to obtain the medical data of their clients. As with any technology, centralized storage of data in a database is vulnerable to hacking or other types of cyber attacks. Therefore, the question of data privacy and security arises also in the field of smart dentistry, as increasingly more data are being obtained from pre-clinical examinations, medical procedures, and treatments. To protect the data from misuse or unauthorized access by third parties, certain security and access control technologies as well as privacy-preserving technologies need to be deployed. Several attempts in research have been made to tackle these issues.

Kayes et al. [45] conducted a survey of context-aware access control mechanisms for cloud and fog networks. Their focus on fog computing is associated with a new access control paradigm to reduce overhead costs by moving the execution of application logic from the center of the cloud data sources to the periphery of IoT-oriented sensor networks. 
They present different taxonomies, such as contextual conditions and authorization models, based on the key issues in this area and discuss the existing context-sensitive access control approaches to tackle the aforementioned issues. They further propose a new generation of Fog-Based Context-Aware Access Control (FB-CAAC) framework, combining the benefits of the cloud, IoT, and context-aware computing and ensuring proper access control and security at the edge of the end devices, which could be perfectly applied to AI devices in dentistry.

Kayes et al. [45] present a context-aware framework for access control. Their framework includes a context model for classifying and capturing access-control-oriented contextual information, a situation model for identifying and defining contextual conditions of concern, and a policy model for specifying context-aware access control policies.

Lien et al. [46] propose a software-embedded method of security protection applied in indirect imaging in dentistry. They stress the problem of the creation of fraudulent secondary capture images that use scanned traditional X-ray films to lower the cost of traditional, digital dental X-ray equipment. The implementation of medical SC images has to conform to security regulations, such as prevention of data modification and authentication of identity, by law. Their proposal suggests a new method, called software-embedded digital signature, to ensure the integrity and authentication of the Digital Imaging and Communications in Medicine (DICOM) standard of SC images scanned from an X-ray film and captured in digital form. The role of AI in dentistry by incorporation of AI in the teaching and learning process using imaging and screening has also been tackled by Majumdar et al. [47].

Lee et al. [48] present a data analytics pipeline for smart healthcare applications to stress the problem caused by the large amount of heterogeneous datasets including images. They present the use of petascale high-performance computing resources to remove temporal and accuracy limitations when processing and analyzing such big data. Their proposed system would dramatically reduce a doctor's workload.

A different approach to tackle the problem of data privacy is chosen by Murrill et al. [49], who discuss the issue of data privacy and cyber security in the context of use of smart meter data. They discuss data privacy and cyber security issues under consideration of different laws, such as the Fourth Amendment, the Stored Communications Act (SCA), the Computer Fraud and Abuse Act (CFAA), the Electronic Communications Privacy Act (ECPA), the Foreign Intelligence Surveillance Act (FISA), the Federal Trade Commission Act (FTC Act), and the Federal Privacy Act of 1974 (FPA).

To summarize our findings, we provide in Table 2 an overview of the dental applications and corresponding technologies used.

Table 2. Overview of dental applications and technologies.

\begin{tabular}{|c|c|c|}
\hline Application & Technology & Author \\
\hline $\begin{array}{l}\text { Caries diagnosis, computer-aided implant dentistry, } \\
\text { digital radiography, electric and surgical/implant } \\
\text { hand pieces, lasers, occlusion, temporomandibular } \\
\text { joint (TMJ) analysis and diagnosis, photography, } \\
\text { practice and patient record management }\end{array}$ & $\begin{array}{l}\text { Computer-assisted design and } \\
\text { computer-assisted manufacture } \\
\text { (CAD/CAM) and intraoral imaging }\end{array}$ & $\begin{array}{l}\text { Spielman [2] } \\
\text { Ahmed et al. [3] }\end{array}$ \\
\hline Pattern recognition in dentistry & $\begin{array}{c}\text { Machine learning, artificial neural } \\
\text { networks (ANNs) }\end{array}$ & $\begin{array}{l}\text { Bindushree et al. [4] } \\
\text { Arora [12] }\end{array}$ \\
\hline Pattern creation for decision making in dentistry & Deep learning & $\begin{array}{c}\text { Marr [13] } \\
\text { Hargrave [14] }\end{array}$ \\
\hline Creation of a mind tool for dentistry & Intelligence augmentation & $\begin{array}{l}\text { Hassani et al. [5] } \\
\text { Cawley [15] }\end{array}$ \\
\hline AI clinical decision support & Bayesian networks & Thanathornwong [19] \\
\hline Treatment outcome analysis & Convolutional neural network & Patcas et al. [20] \\
\hline
\end{tabular}


Table 2. Cont.

\begin{tabular}{|c|c|c|}
\hline Application & Technology & Author \\
\hline Social properties and attributions of robots & Internet of Things (IoT) & Dhelim et al. [25] \\
\hline Patient management and treatment plans & $\begin{array}{c}\text { Practice intelligence platform, computer } \\
\text { vision software }\end{array}$ & Hellopearl [28] \\
\hline $\begin{array}{l}\text { AI training with radiographic data of } \\
\text { common pathologies }\end{array}$ & AI-empowered radiograph diagnostic & $\begin{array}{l}\text { Hellopearl [28] } \\
\text { Denti [29] }\end{array}$ \\
\hline Oral-cancer-screening device & Visual enhanced lesion (VEL) scope & Dentistry iQ [30] \\
\hline Dental caries diagnostics & DIAGOdent laser system & Dentistry iQ [30] \\
\hline $\begin{array}{l}\text { Dental fear, dental anxiety, dentist phobia, } \\
\text { odontophobia, or dentophobia }\end{array}$ & Air abrasion & Gentle Dental [34] \\
\hline Precise physiological reinstation of occlusion & Functioning masticatory system & Agbaje et al. [37] \\
\hline Intraoral scanner & Planmeca Emerald & \\
\hline Effectiveness of the brushing method & AI tooth brushes & Peters [42] \\
\hline $\begin{array}{l}\text { Context-aware access control mechanisms for cloud } \\
\text { and fog networks }\end{array}$ & AI devices in dentistry & Kayes et al. [45] \\
\hline Simulation of fatigue and gag reflex & Dental patient robot & Das [38] \\
\hline $\begin{array}{l}\text { Automatic probing, drilling, cleaning, shaping, and } \\
\text { filling of the root canal }\end{array}$ & Endo micro-robot & Bhat [40] \\
\hline
\end{tabular}

\section{Prospects and Investment}

The value of AI employed in the global healthcare market is expected to reach USD 45.2 billion by 2026 from USD 4.9 billion in 2020. In other words, about USD 40 billion growth in the next 6 years is anticipated. AI is projected to grow at a compound annual growth rate (CAGR) of $44.9 \%$ during the forecast period. Multiple factors are prompting this rise, the most important of which include the following:

- The increasing volume and complexities of healthcare datasets, the obvious need to decrease ascending healthcare costs, and the spreading of inter-industrial cooperation. One of the factors whose role in boosting the market growth of AI has recently been highlighted is that multiple pharmaceutical and biotechnology companies worldwide are adopting AI technologies to speed up COVID-19 vaccine or drug development processes.

- Evidence exists that an originative inter-professional coordination among clinicians, researchers, and engineers will lead to further AI development in dentistry.

- $\mathrm{ML}$ in the AI healthcare market is projected to grow at the highest CAGR during the next decade.

- The United States and Canada hold the lion's share, with 73\% of the total VC capital directed toward health startups and AI and health receiving $62 \%$ of such investments. Europe follows with much smaller figures: $14.40 \%$ of total VC investments concerning health startups and $19.33 \%$ of these investments going to $\mathrm{AI}$ and health startup companies.

- The potential to increase the usage of deep learning in various healthcare programs, especially in medical imaging, disease diagnosis, and drug discovery, as well as the use of sensors and devices to track a patient's health status in real time, complements market growth. A clear understanding of AI techniques and concepts certainly has an advantage to facilitate deployment of AI technologies in dentistry in the near future. There are also hopes that AI will soon be fully applied in orthodontics, endodontics, and restorative dentistry.

Currently, a major limitation for further deployment of AI in dentistry is the lack of sufficient and accurate data. Therefore, it is currently the responsibility of dentists and 
physicians to focus on collecting and entering valid data into their database so that it can be fully used for AI in dentistry in the future.

\section{Obstacles and Limitations of Robotic Dentistry}

According to Frey and Osborne (2013) from the Oxford Martin School, in their publication "The Future of Employment: How Susceptible Are Jobs to Computerization?," there is almost no chance for dentists to be replaced by robots [43]. This seems quite reasonable. Although AI has played a special part in helping the industry to grow, biological structures are much more complicated and replacing human knowledge, decision-making ability, and many more human-specific capabilities is not an easily attainable purpose. While robots are rapidly evolving in mimicking human behavioral patterns and moods, they are not able to perceive and anticipate exactly how someone feels. Empathy is one of the main characteristics that is not expected to be seen in robots. Hence challenges both in technical and in ethical aspects still exist [44].

Dental professionals are hesitant to adopt AI-based technologies. An alternative solution that brings together the capabilities of technology and the dentist reconciling both $\mathrm{AI}$ and human features is preferred so that the process of data collection and categorization be trouble free and in spite of sustaining the human aspects of clinical care [44].

Finally, as the creator of intelligent machines to simulate the human brain and nervous system, humanity is not yet fully informed about all brain and body functions. Therefore, it is not rational to think that artificial artifacts may get there so soon. Even if one day we can finally bring the complexity of robots closer to human brains, the issue of cost arises, which is one of the main obstacles to reaching a fully robotic industry. Adapting the global dental industry to the use of machines requires a huge investment, especially in the initial stages, which, if not provided, will lead to the dismantling of this project [3].

\section{Future Research Issues}

Potential future research in the area of $\mathrm{AI}$ in dentistry may take a closer look at the various obstacles and challenges associated with the deployment of AI methods in dentistry, as well as upcoming future technologies applicable to AI. Potential topics may comprise the legal challenges associated with liability questions in the case of malfunctions or accidents occurring during diagnosis, treatment, or post-treatment. Furthermore, economic studies could focus on the economies-of-scale effect, given the rapid growth in deployment rates in dentistry. Associated with this study, new methodologies or treatment applications might arise out of the research, with the effect of lowering deployment costs and, thus, benefiting patients and public health insurance companies.

New advances in technology, e.g., emerging out of neuroscience might link humansensor interfaces with robots or nano-robots, which might provide excellent non-invasive treatments, which otherwise might be a risk for the patient. Prospective research could tackle the areas of neuroscience and nano-science.

Nevertheless, traditional AI research topics are still in progress, such as image processing and pattern recognition. Advances in these areas would mean a tremendous advance in the quality of pre-diagnostics and assembling, resp. upscaling, of data quality used for image processing. Advances in data quality would result in a significant increase in treatment quality.

In the area of philosophy, trans-humanism is an emerging field of study. Linked to the application of $\mathrm{AI}$ in dentistry, the philosophical questions arising out of questions about the boundaries and potential threats of AI applied to treatment of the human body could be challenged.

\section{Conclusions}

In this article, we discussed the types of $\mathrm{AI}$ and the effects they have had on the work of dentists and dental students so far, as well as the direction that the implementation of AI technologies will take in the future. Existing studies, including our overview of 
AI technologies, show that over time, dentistry, like all other disciplines and sciences, is advancing rapidly, with the role of AI and technologies contributing significantly to these advances.

Currently, AI has entered various areas of dentistry and has provided significant services in each of these areas. However, in many areas, there have been interesting but failed efforts and ideas that, with further progress being made in using the potentials of AI. Going into the phase of investment in AI projects in dentistry can lead to facilitation of their deployment and to new developments of efficiency in the field of dentistry. However, a still unclear question is about the impacts of $\mathrm{AI}$ in dentistry on employment in this sector and whether AI technologies will be replacing humans or whether they will be rather increasing their efficiency. According to our background review of existing dentistry AI technologies, we assume that different processes in the dental profession are largely multidimensional and require various subtleties in different areas. By looking at the types of subsets of AI and spectating their progress, it seems that $\mathrm{AI}$ is at the service of the dentists to augment their intelligence and efficiency, rather than being a threat for employment processes in the dentistry sector.

Author Contributions: Conceptualization, H.H., K.N., A.G., P.A.A., N.K., and S.U.; investigation, H.H., K.N., A.G., P.A.A., N.K., and S.U.; writing-review and editing, H.H., K.N., A.G., P.A.A., N.K., and S.U. All authors have read and agreed to the published version of the manuscript.

Funding: This research received no external funding.

Conflicts of Interest: The authors declare no conflict of interest.

\section{References}

1. FDI. World Dental Federation. Facts, Figures and Stats-Oral Disease: 10 Key Facts. Available online: https://www. fdiworlddental.org/oral-health/ask-the-dentist/facts-figures-and-stats (accessed on 23 December 2020).

2. Spielman, A.I. Technology in Dentistry, Through the Ages. NYU. Available online: https:// dental.nyu.edu/aboutus/history-ofnyucd/technology-in-dentistry-through-the-ages.html (accessed on 23 December 2020).

3. Ahmed, M.S.; Chaturya, K.; Tiwari RV, C.; Virk, I.; Gulia, S.K.; Pandey, P.R.; Tiwari, H. Digital Dentistry-New Era in Dentistry. J. Adv. Med Dent. Sci. Res. 2020, 8, 67-70.

4. Bindushree, V.; Sameen, R.J; Vasudevan, V.; Shrihari, T.G.; Devaraju, D.; Mathew, N.S. Artificial intelligence: In modern dentistry. J. Dent. Res. Rev. 2020, 7, 27.

5. Hassani, H.; Silva, E.S.; Unger, S.; TajMazinani, M.; Mac Feely, S. Artificial Intelligence (AI) or Intelligence Augmentation (IA): What Is the Future? AI 2020, 1, 143-155. [CrossRef]

6. Science Daily. Artificial Intelligence. Available online: https://www.sciencedaily.com/terms/artificial_intelligence.htm (accessed on 23 December 2020).

7. Sternberg, R.J. Human Intelligence. Encyclopedia Britannica. Available online: https:/ /www.britannica.com/science/humanintelligence-psychology (accessed on 23 December 2020).

8. Chen, Y.W.; Stanley, K.; Att, W. Artificial intelligence in dentistry: Current applications and future perspectives. Quintessence Int. 2020, 51, 248-257.

9. Available online: https://www.planmeca.com/cadcam/dental-scanning/planmeca-emerald/ (accessed on 29 August 2021).

10. Whelton, H.; Ramsdale, M.P.; Zormpas, E. Extracting Value from Big Data in Oral Health Care Services. In Dental Outlook Special Issue for the 23rd General Meeting of the Japanese Association for Dental Science, Fukuoka, Japan, 21-23 October 2016; Ishiyaku Publishers: Tokyo, Japan, 2017; pp. 130-133.

11. Available online: https://hellopearl.com/insights\#section2 (accessed on 29 August 2021).

12. Arora, S. 5 Major Differences between Machine Learning and Deep Learning. Simplilearn. Available online: https: //www.simplilearn.com/machine-learning-vs-deep-learning-major-differences-you-need-to-know-article (accessed on 23 December 2020).

13. Marr, B. Forbes. 10 Amazing Examples of How Deep Learning AI Is Used in Practice. Available online: https:/ /www.forbes.com/ sites/bernardmarr/2018/08/20/10-amazing-examples-of-how-deep-learning-ai-is-used-in-practice/?sh=10124dcef98a (accessed on 23 December 2020).

14. Hargrave, M. Investopedia. Deep Learning. Available online: https://www.investopedia.com/terms/d/deep-learning.asp\# what-is-deep-learning (accessed on 23 December 2020).

15. Cawley, C. Tech.Co. Why Artificial Intelligence Will Never Compete with the Human Brain. Available online: https://tech.co/ news/artificial-intelligence-human-brain-2018-04 (accessed on 23 December 2020).

16. Kavo. DEXvoice-The Smart Solution for Your Dental Workflow. Available online: https://www.kavo.com/en-us/dexvoicesmart-solution-your-dental-workflow (accessed on 23 December 2020). 
17. Lee, J.H.; Kim, D.H.; Jeong, S.N.; Choi, S.H. Detection and diagnosis of dental caries using a deep learning-based convolutional neural network algorithm. J. Dent. 2018, 77, 106-111. [CrossRef]

18. Jung, S.-K.; Kim, T.-W. New approach for the diagnosis of extractions with neural network machine learning. Am. J. Orthod Dentofac. Orthop. 2016, 149, 127-133. [CrossRef]

19. Thanathornwong, B. Bayesian-based decision support system for assessing the needs for orthodontic treatment. Health Inf. Res. 2018, 24, 22-28. [CrossRef]

20. Patcas, R.; Bernini, D.A.J.; Volokitin, A.; Agustsson, E.; Rothe, R.; Timofte, R. Applying artificial intelligence to assess the impact of orthognathic treatment on facial attractiveness and estimated age. Int. J. Oral Maxillofac. Surg. 2019, 48, 77-83. [CrossRef] [PubMed]

21. Feres, M.; Louzoun, Y.; Haber, S.; Faveri, M.; Figueiredo, L.C.; Levin, L. Support vector machine-based differentiation between aggressive and chronic periodontitis using microbial profiles. Int. Dent. J. 2018, 68, 39-46. [CrossRef]

22. Kim, D.W.; Kim, H.; Nam, W.; Kim, H.J.; Cha, I.H. Machine learning to predict the occurrence of bisphosphonate-related osteonecrosis of the jaw associated with dental extraction: A preliminary report. Bones 2018, 116, 207-214. [CrossRef] [PubMed]

23. Speight, P.; Elliott, A.; Jullien, J.; Downer, M.; Zakzrewska, J. The use of artificial intelligence to identify people at risk of oral cancer and precancer. Br. Den. J. 1995, 170, 382-387. [CrossRef] [PubMed]

24. Schwendicke, F.; Samek, W.; Krois, J. Artificial Intelligence in Dentistry: Chances and Challenges. J. Dent. Res. 2020, 99, 769-774 [CrossRef]

25. Dhelim, S.; Ning, H.; Farha, F.; Chen, L.; Atzori, L.; Daneshmand, M. IoT-Enabled Social Relationships Meet Artificial Social Intelligence. IEEE Internet Things J. 2021. [CrossRef]

26. Cai, X.; Ning, H.; Dhelim, S.; Zhou, R.; Zhang, T.; Xu, Y.; Wan, Y. Robot and its living space: A roadmap for robot development based on the view of living space. Digit. Commun. Netw. 2020. [CrossRef]

27. Byrne, J. Personalized medicine in dentistry-Is genotyping the "next frontier" of periodontitis treatment? Inside Dent. 2013, 9. Available online: https:/ / www.aegisdentalnetwork.com/id/2013/10/personalized-medicine-dentistry (accessed on 23 December 2020).

28. Hellopearl. Technology Solutions that Put Dentists and Patients at ease. Available online: https://hellopearl.com/products (accessed on 23 December 2020).

29. Denti.AI. Automated Diagnostics for X-ray Images in Dentistry. Available online: https://www.denti.ai/ (accessed on 23 December 2020).

30. Dentistry iQ. VEL Scope Is a Non-Invasive Oral Cancer-Screening Device. Available online: https://www.dentistryiq.com/ dentistry/oral-cancer/article/16362585/velscope-is-a-noninvasive-oral-cancerscreening-device (accessed on 23 December 2020).

31. Cicciù, M.; Cervino, G.; Fiorillo, L.; D’Amico, C.; Oteri, G.; Troiano, G.; Zhurakivska, K.; Lo Muzio, L.; Herford, A.S.; Crimi, S.; et al. Early diagnosis on oral and potentially oral malignant lesions: A systematic review on the VELscope ${ }^{\circledR}$ Fluorescence method. Dent. J. 2019, 7, 93. [CrossRef] [PubMed]

32. Dentistry iQ. Diagnodent Caries Detection Aid. Available online: https:/ / www.dentistryiq.com/dentistry/restorative-cosmeticand-whitening/article/16359316/diagnodent-caries-detection-aid (accessed on 23 December 2020).

33. The Guardian. Scared of the Dentist? This Is Why, Say Neuroscientists. Available online: https://www.theguardian.com/society/ 2013/nov/10/dentists-drills-brain-neuroscientists (accessed on 23 December 2020).

34. Gentle Dental. Overcoming Dentophobia, a Fear of the Dentist. Available online: https://www.gentledental-mi.com/overcomingdentophobia-a-fear-of-the-dentist/ (accessed on 23 December 2020).

35. Westermeier Martin. What Is Air Abrasion? Available online: https://wmsmile.com/what-is-air-abrasion/ (accessed on 23 December 2020).

36. Hegde, V.S.; Khatavkar, R.A. A new dimension to conservative dentistry: Air abrasion. J. Conserv. Dent. 2010, 13, 4. [CrossRef]

37. Agbaje, J.O.; Van de Casteele, E.; Salem, A.S.; Anumendem, D.; Shaheen, E.; Sun, Y.; Politis, C. Assessment of occlusion with the T-Scan system in patients undergoing orthognathic surgery. Sci. Rep. 2017, 7, 1-8. [CrossRef]

38. Das, H. Robotics in Dentistry: The Next Generation Technology. Dental Reach. Available online: https://dentalreach.today/ dental-education/robotics-in-dentistry-the-next-generation-technology/ (accessed on 23 December 2020).

39. SIMROID. MORITA. Simroid: Patient Simulation System for Dental Education. Available online: https://www.morita.com/group/ en/products/educational-and-training-systems/training-simulation-system/simroid/?tab=features (accessed on 23 December 2020).

40. Bhat, B.D.; Bhandary, S.; Naik, R.; Shetty, D. Robotics in dentistry: Fiction or reality. J. Dent. Res. Rev. $2017,4,67$.

41. Webb, A.; Sommers, S. Dentistry iQ. Innovations in Dentistry: Navigational Surgery, Robotics, and Nanotechnology. Available online: https:/ / www.dentistryiq.com/dentistry/article/14183983/innovations-in-dentistry-navigational-surgery-robotics-andnanotechnology (accessed on 23 December 2020).

42. Peters, J. The Verge. Oral-B’s New $\$ 220$ Toothbrush Has AI to Tell You When You're Brushing Poorly. Available online: https: //www.theverge.com/ circuitbreaker/2019/10/25/20932250/oral-b-genius-X-connected-toothbrush-ai-artificial-intelligence (accessed on 23 December 2020).

43. Frey, C.B.; Osborne, M.A. The future of employment: How susceptible are jobs to computerisation? Technol. Forecast. Soc. Chang. 2017, 114, 254-280. [CrossRef]

44. Tandon, D.; Rajawat, J. Present and future of artificial intelligence in dentistry. J. Oral Biol. Craniofacial Res. 2020, 10, 391-396. [CrossRef] [PubMed] 
45. Kayes, A.S.; Kalaria, R.; Sarker, I.H.; Islam, M.; Watters, P.A.; Ng, A.; Hammoudeh, M.; Badsha, S.; Kumara, I. A Survey of Context-Aware Access Control Mechanisms for Cloud and Fog Networks: Taxonomy and Open Research Issues. Sensors 2020, 20, 2464. [CrossRef]

46. Lien, C.Y.; Kao, T.; Hsiao, C.H.; Keng, C.I. A Software-embedded Method of Security Protection Applied in Indirect Imaging in Dentistry. J. Med. Biol. Eng. 2010, 30, 203-207. [CrossRef]

47. Majumdar, B.; Sarode, S.C.; Sarode, G.S.; Patil, S. Technology: Artificial intelligence. BDJ 2018, 224, 916. [CrossRef]

48. Lee, C.; Murata, S.; Ishigaki, K.; Date, S. A Data Analytics Pipeline for Smart Healthcare Applications. In Sustained Simulation Performance; Springer: Cham, Switzerland, 2017. [CrossRef]

49. Murrill, B.J.; Liu, E.C.; Thompson, R.M. Smart Meter Data: Privacy and Cybersecurity. In Privacy: Select Issues and Laws for the 21st Century; Nova: Hauppauge, NY, USA, 2013; pp. 1-45. 